$\xi^{2}=-$

\title{
Energy Efficiency Optimization in Cooperative Spectrum Sensing by NOMA
}

\author{
Meenakshi Awasthi ${ }^{1 *}$, Madhav J. Nigam² ${ }^{2}$ Vijay Kumar ${ }^{3}$ \\ ${ }^{1}$ Research Scholar, ${ }^{2}$ Professor, ${ }^{3}$ Associate Professor, \\ ${ }^{1,2,3}$ ECE Department, IIT Roorkee, Roorkee, India

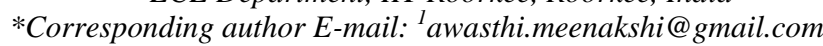

\begin{abstract}
Energy efficiency improvement is a major point of concern in cooperative scenario of cognitive radio networks, so as to compensate the overhead occurred due to power consumed by multiple numbers of secondary users. Non-orthogonal multiple access (NOMA) is a potential technique to get the higher spectrum efficiency for $5 \mathrm{G}$ systems. The basic feature of NOMA is to utilize the same channel, time and code but at different power levels, which results in higher spectrum efficiency (SE). In this article, firstly the cognitive radio (CR) inspired NOMA is generalized by multiple primary users. Then, energy efficiency (EE) is optimized for the proposed system. An efficient algorithm is proposed for the non-convex fractional programming problem. Simulation results show the superior performance over conventional multiple accesstechniques.
\end{abstract}

Keywords: Cognitive radio, Non-orthogonal multiple access (NOMA), Cooperative NOMA, Energy efficiency.

\section{Introduction}

Non-orthogonal multiple access has emerged as the promising technique to improve SE of future $5 \mathrm{G}$ communication systems [1]-[3]. In comparison to conventional multiple access (CMA) techniques, NOMA is empowered by the superposition coding, distinct power allocation and successive cancellation of interference [4]. That is why it has been emerged as more spectral efficient technique for future 5G systems. In third Generation Partnership Project Long Term Evolution (3Gpp-LTE)NOMA was applied for downlink system [5]. The application of NOMA has been illustrated in [6]-[8].

Moreover, cognitive radio is another approach to improve SE, proposed by [9], [10]. In CR networks multiple secondary users (SUs) opportunistically utilize the licensed or primary users (PUs) spectrum by spectrum sensing, detection and allocation. Initially, NOMA inspired CR has been proposed by[4], [7], [11].

\section{Related work}

The research in [4] indicates that within multiple users, a user with better channel will be a secondary user and poorer channel as primary user. The secondary user will try to occupy the channel of the primary user. Further, NOMA for underlay cognitive radio networks of large scale was also applied in [11].

Apart from SE, EE is also drawing the attention of researchers as communication technology is responsible for $5 \%$ of the tota world energy consumption [12]. As of now, very few researches are available in the field of NOMA with respect to EE in cognitive radio networks [13]. In [14],[15] author proposed EE optimization for fading MIMO inspired by non-orthogonal multiple access.
The aforementioned observations, leads us to motivate the study of EE improvement in NOMA inspired CRNs.

The existing CR-NOMA proposed in [4] is generalized by considering one base station (BS), one PU and SU. A user, associated with strong channel is regarded as SU and PU with weak channel conditions. This unlicensed SU can take on the spectrum with the condition of less interference to PU. The main objective of this paper is to maximize the $\mathrm{EE}$ with the constraint of quality of service of PU or the licensed user. Here, the problem is formulated as the non- convex fractional programming problem.

Further in the paper, system model is briefed in section 3. In section 4, problem formulation and solution is given. Section 5 illustrated the simulation results. Finally, the conclusion is discussed in section 6 .

\section{System model}

In the system model, downlink transmission with one BS with two antennas, working simultaneously for two users (PU and SU) is considered. Both the users are equipped with antennas. Let the channel between PU and BS \& SU and BS is flat with quasi-static fading. In this research, is it assumed that SU has better channel as compared to PU, hence it access the PU spectrum by NOMA with the constraint of non degradation of PU performance. The user 1 is $\mathrm{PU}$ and user 2 is SU. For a generalized CR-NOMA framework, the $\mathrm{M}$ users are served by a single BS simultaneously inspired by NOMA with the condition of providing quality of service to rest M-1 PU.

Let the BS transmits the message $x_{1}$ to $\mathrm{PU}$ and $x_{2}$ to $\mathrm{SU}$ at the same frequency and same time slot but different power level. The BS is transmitting the superposition of weighted symbols $\left\{w_{M} x_{M}\right\}_{M=1}^{2}$. The received signal is a combination of the desired message plus interference plus additive noise. Mathematically, the 
observations received at the PU and SUcan be represented, respectively as

$$
\begin{array}{r}
y_{1}=h_{1}^{H} w_{1} x_{1}+h_{1}^{H} w_{2} x_{2}+n_{1} \\
y_{2}=h_{2}^{H} w_{2} x_{2}+h_{2}^{H} w_{1} x_{1}+n_{2}
\end{array}
$$

where, the first component of equation (1) is message, second is interference produced by the other message and the third one is noise, $n_{1}$ and $n_{2}$ are additive Gaussian noise with zero mean and variance $\sigma^{2}$. The channel vector, $h_{M}$ can be represented as $h_{M}=$ $g_{M} d_{M}^{\frac{-a}{2}}$, where $g_{M} \sim \mathrm{CN}\left(0, I_{2}\right), d_{M}$ is distance between BS and the $M^{\text {th }}$ user, and $a$ is the path loss exponent.

Further to implement NOMA in CRNs scenario, successive interference cancellation (SIC) is used so that the partial interference can be removed[14], [16]. Here, we have assumed that PU is far from the BS while SU isnear to BS hence SU is strong user and PU is weak. In SIC decoding technique, PU will decode the message $x_{1}$ by taking SU interference as noise. While SU will first decode the message $x_{1}$ treating the interference by $x_{2}$ as noise then cancel the $x_{1}$ part from the received one. Thus, it will decode the $x_{2}$ from the remaining message. The user's decoding ability is given by its signal to interference - noise ratio (SINR)[17]. The general form of representation of SINR when, $M=1,2$ and $1 \leq \mathrm{i} \leq$ $M$ isgiven by

$\operatorname{SINR}_{M}^{i}=\frac{\left|h_{M}^{H} w_{i}\right|^{2}}{\sum_{j=i+1}^{2}\left|h_{M}^{H} w_{j}\right|^{2}+\sigma^{2}}$

The above condition will be true, provided SU will decode the messages intended for the PU.

\section{Problem Formulation and Solution}

\subsection{EE Maximization Problems}

Here, the EE maximization problem is formed as the ratio of maximum achievable sum rate and total power consumed and represented as

$$
E E \triangleq \frac{R_{t}}{P_{t}+P_{c}}
$$

where, $R_{t}$ is the sum rate in bits/sec/Hz, transmit power $P_{t} \triangleq$ $\sum_{M=1}^{2}\left\|w_{M}\right\|_{2}^{2}$ and $P_{c}$ circuit consumption power. The total achievable sum rate is the sum of PU rate and SU rate. The PU sum rate is given by

$R_{P U} \triangleq \log _{2}\left(1+L_{1}\right)$

where, $L_{1}$ is the SINR threshold for the decoding of $x_{1}$.

In the SU decoding, SIC and predefined order will be used so that interference due to other user could be removed. Hence, the SU sum rate is formulated as

$R_{S U} \triangleq \log _{2}\left(1+\frac{\left|h_{2}^{H} w_{2}\right|^{2}}{\sigma^{2}}\right)$

Accordingly, the problem of EE maximization can be formulated as

$$
\begin{aligned}
& \max _{w_{1}, w_{2}} E E \\
& \text { s.t. } S I N R_{1}^{1} \geq L_{1}, M=1 \\
& \sum_{M=1}^{2}\left\|w_{M}\right\|_{2}^{2} \leq P_{\text {tot }}
\end{aligned}
$$

Here, the first constraint will give the QoS required for successfu transmission of PU and the second constraint will give the transmit power requirement. The problem defined in equation (7) is a non-convex fractional programming problem.

\subsection{Proposed Algorithm}

The problem defined in (6) is further explored by putting the values of SINR and is given as

$$
\begin{aligned}
& \max _{w_{1}, w_{2}} E E \\
& \text { s.t. }\left|h_{1}^{H} w_{1}\right|^{2} \geq L_{1}\left(\left|h_{1}^{H} w_{2}\right|^{2}+\sigma^{2}\right), M=1 \\
& \left|h_{2}^{H} w_{1}\right|^{2} \geq L_{1}\left(\left|h_{2}^{H} w_{2}\right|^{2}+\sigma^{2}\right), M=2 \\
& \sum_{M=1}^{2}\left\|w_{M}\right\|_{2}^{2} \leq P_{\text {tot }}
\end{aligned}
$$

The above problem given in (7) is non-convex problem. This type of non - convex fractional programming problem can be solved by the Dinkelbach's algorithm[18].But the computational complexity of this algorithm is very high. Hence, convex approximation is used to solve this problem. Further to explore the hidden convexity, the problem (7)can be rewritten in term of auxiliary variabless and $u$ as

$$
\begin{array}{ll} 
& \max _{w_{1}, w_{2}, s, u} s \\
\text { s.t. } R_{P U}+R_{S U} \geq \sqrt{s u} & \\
P_{t}+P_{c} \leq \sqrt{u} &
\end{array}
$$

If $v$ is taken as auxiliary variable which is used to indicate the target SINR for the $M^{\text {th }}$ user and $r$ is the data rate of $v$ (data rate of $\mathrm{SU})$, then $\frac{\left|h_{M}^{H} w_{M}\right|^{2}}{\sigma^{2}} \geq v$, and $1+v \geq 2^{r}$. The squared EE can be interpreted by the $s$ and squared power can be termed as $u$. With the explanations given above the original fractional programming problem can be approximated as convex by using Taylor's approximation with iterations and can be formulated for $n^{\text {th }}$ iteration as

s. t. $\frac{y T\left(\emptyset_{M, M}, \phi_{M, M}^{n}\right)}{\sigma^{2}} \geq v$

$$
\max _{w_{1}, w_{2}, s, u, v, r} S
$$

where, $\quad\left|h_{M}^{H} w_{i}\right|^{2}={\emptyset_{R}}^{2}+\emptyset_{I}{ }^{2}, \quad \emptyset_{M, i} \triangleq\left[\emptyset_{M, i}{ }^{R}+\emptyset_{M, i}{ }^{I}\right]^{T}$ and $y\left(\emptyset_{M, i}\right)$ is Euclidian norm of real vector. $y T\left(\emptyset_{M, M}, \emptyset_{M, M}^{n}\right)$ is $1^{\text {st }}$ order- Taylor's approximation. Considering this, the approximations of the constraints are

$$
\begin{aligned}
& \quad y T\left(\emptyset_{M, M}, \emptyset_{M, M}^{n}\right) \geq L_{1}\left(\left|h_{1}^{H} w_{2}\right|^{2}+\sigma^{2}\right), \quad M=1 \\
& y T\left(\emptyset_{M, i}, \emptyset_{M, i}^{n}\right) \geq L_{1}\left(\left|h_{2}^{H} w_{2}\right|^{2}+\sigma^{2}\right), M=2 \\
& \emptyset_{M, i}^{n} \triangleq\left[\operatorname{Re}\left\{\left(h_{M}^{H} w_{i}\right)^{n-1}\right\}, \operatorname{Im}\left\{\left(h_{M}^{H} w_{i}\right)^{n-1}\right\}\right]^{T}
\end{aligned}
$$

$u^{(n)}=\left(\sum_{M=1}^{2}\left\|w_{M}^{n-1}\right\|_{2}^{2}+P_{c}\right)^{2} \quad(12) s^{(n)}=\frac{\left\{R_{P U}+\log _{2}\left(1+\frac{\left|h_{2}^{H} w_{2}\right|^{2}}{\sigma^{2}}\right\}^{2}\right.}{u^{(n)}}$ (13)

The proposed algorithm shown in Algorithm1, gives the EE optimization method. Firstly, the initial values of $\left\{\mathrm{w}_{\mathrm{M}}^{(0)}\right\}_{\mathrm{M}=1}^{2}$, $s^{(0)}, u^{(0)}, v^{(0)}, r^{(0)}$ are taken. Then, equation (9) is solved for $\mathrm{n}=0$. In step 5, update $\mathrm{n}=\mathrm{n}+1$ and then solve the equations (11), (12), and (13) for the updated value of n. Lastly, the loop is terminated when reach the convergence. Further, the optimized values are obtained.

\section{Algorithm 1 CR NOMA for EE optimization}

$\begin{array}{ll}\text { 1. } & \text { Initialization: }\left\{\mathrm{w}_{\mathrm{M}}^{(0)}\right\}_{\mathrm{M}=1}^{2}, s^{(0)}, u^{(0)}, v^{(0)}, r^{(0)} . \\ \text { 2. } & \mathrm{n}=0 . \\ \text { 3. } & \text { repeat } \\ \text { 4. } & \text { solve the equation }(9) . \\ \text { 5. } & \mathrm{n}=\mathrm{n}+1 . \\ 6 . & \text { solve equation }(11),(12) \text { and }(13) \text { for updated } \mathrm{n} . \\ \text { 7. } & \text { stop when reach the convergence. }\end{array}$

\section{Simulation Results}

The performance of the system has been illustrated in this section by comparing the NOMA with the conventional TDMA tech- 
nique. The simulation parameters used for MATLAB are given in table1:

Table 1: Simulation parameters

\begin{tabular}{|c|c|c|c|c|c|c|c|}
\hline Parameters & $d$ & $f_{s}$ & $a$ & $\sigma^{2}$ & $P_{c}$ & $d_{l}$ & $R_{P U}$ \\
\hline Values & 40 & 300000 & 3.5 & -80 & 30 & 50 & 2 \\
\hline & $\mathrm{m}$ & $0 \mathrm{~Hz}$ & & $\begin{array}{c}\mathrm{dB} \\
\mathrm{m}\end{array}$ & $\begin{array}{c}\text { Wat } \\
\mathrm{t}\end{array}$ & $\mathrm{m}$ & $\begin{array}{c}\text { bits/Hz } \\
\text { s }\end{array}$ \\
\hline
\end{tabular}

In figure 1, EE versus transmission power is illustrated for NOMA and TDMA. Moreover, the comparison of NOMA and conventional TDMA (used in exhaustive search method and sub-optimal algorithm in []) is also presented. Figure 1 shows the superior performance of the NOMA technique over the conventional TDMA in terms of EE. At the transmission power $0.2 \mathrm{Watt}$, the EE for NOMA is $0.998 \mathrm{bits} / \mathrm{Hz} / \mathrm{J}$ oule, whereas $0.6 \mathrm{bits} / \mathrm{Hz} / \mathrm{J}$ oule for sub-optimal. It is clearly visible from the graph that an increase in transmission power results in decrease of EE. However, the maximum EE for NOMA achieved at 0.1 Watt power.

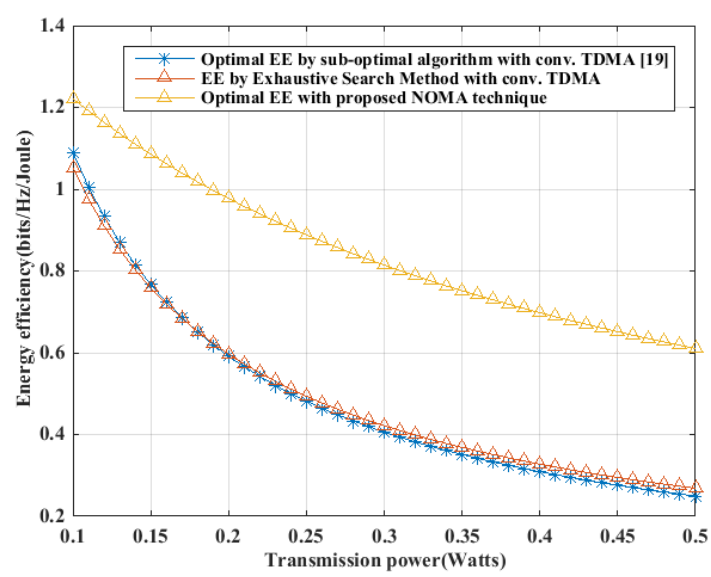

Fig. 1: EE versus transmission power.

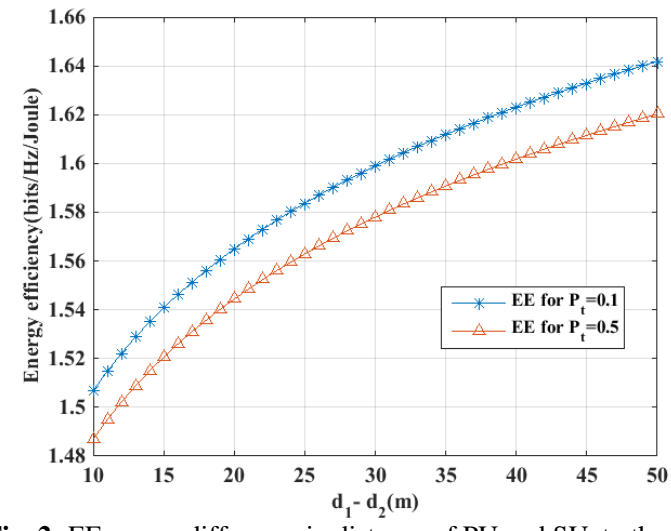

Fig. 2: EE versus difference in distance of PU and SU, to the BS

Figure 2 shows the impact of location of PU and SU from BS on EE. Here, it is assumed that the $d_{1}$ is fixed and $d_{2}$ is varying. Both the distances are calculated from the BS. It is depicted in the figure2 that increase in the difference of distance results in increase in EE. It means, SU is going closer to the BS as difference is increasing. The important conclusion from the figure is, near -fareffect between PU and SU gives more benefit to the NOMA technique, which further increase its applications.

\section{Conclusions}

In this paper, the EE maximization problem with downlink NOMA technique is studied. Here, BS equipped with two antennas, one PU and one SU have considered. Application of NOMA technique in cognitive radio is discussed. CR-NOMA technique will further increase the application of CR into 5G systems. Further, the EE is maximized for the NOMA and compared with otherexisting conventional multiplexing techniques. Results reveal the superior performance of the proposed method. EE maximization problem for multiple PU and multiple SU with MIMO for different fading channels can be considered as future scope.

\section{References}

[1] Y. Saito, Y. Kishiyama, A. Benjebbour, T. Nakamura, A. Li, and K. Higuchi, "Non-orthogonal multiple access (NOMA) for cellular future radio access," IEEE Veh. Technol. Conf., pp. 0-4, 2013.

[2] J. Choi, "Non-orthogonal multiple access in downlink coordinated two-point systems," IEEE Commun. Lett., vol. 18, no. 2, pp. 313316, 2014.

[3] Z. Ding, Z. Yang, P. Fan, and H. V. Poor, "On the performance of non-orthogonal multiple access in 5G systems with randomly deployed users," IEEE Signal Process. Lett., vol. 21, no. 12, pp. 1501-1505, 2014.

[4] Z. Ding, P. Fan, and H. V. Poor, "Impact of User Pairing on 5G Nonorthogonal Multiple-Access Downlink Transmissions," IEEE Trans. Veh. Technol., vol. 65, no. 8, pp. 6010-6023, 2016.

[5] L. Lv, Q. Ni, Z. Ding, and J. Chen, "Application of NonOrthogonal Multiple Access in Cooperative Spectrum-Sharing Networks Over Nakagami- \$m\$ Fading Channels," IEEE Trans. Veh. Technol., vol. 66, no. 6, pp. 5506-5511, 2017.

[6] Z. Ding, Y. Liu, J. Choi, Q. Sun, M. Elkashlan, and H. V. Poor, "Application of Non-orthogonal Multiple Access in LTE and 5G Networks Application of Non-orthogonal Multiple Access in LTE and 5G Networks," IEEE Commun. Mag., vol. 55, no. 2, pp. 185 $191,2017$.

[7] N. Nandan, S. Majhi, and H. Wu, "Secure Beamforming for MIMO-NOMA Based Cognitive Radio Network," vol. 7798, pp. $1-$ 4, 2018.

[8] E. Q. Frp et al., "NOMA:From concept to standardization," 2015 IEEE Conf. Stand. Commun. Netw., vol. 8, pp. 18-23, 2015.

[9] J. Mitola, "Cognitive radio for flexible mobile multimedia communications,” Mob. Multimed. Commun. 1999. (MoMuC '99) 1999 IEEE Int. Work., vol. 22102, pp. 3-10, 1999.

[10] S. Haykin, "Cognitive radio: Brain-empowered wireless communications," IEEE J. Sel. Areas Commun., vol. 23, no. 2, pp. 201-220, 2005.

[11] Y. Liu and S. Member, "Nonorthogonal multiple access in largescale underlay cognitive radio netwroks," IEEE Trans. Veh. Technol., vol. 65, no. 12, pp. 10152-10157, 2016.

[12] Y. Zhang, H. M. Wang, T. X. Zheng, and Q. Yang, "EnergyEfficient Transmission Design in Non-orthogonal Multiple Access," IEEE Trans. Veh. Technol., vol. 66, no. 3, pp. 2852-2857, 2017.

[13] L. Lv, J. Chen, Q. Ni, and Z. Ding, "Design of cooperative nonorthogonal multicast cognitive multiple access for $5 \mathrm{G}$ systems: User scheduling and performance analysis," IEEE Trans. Commun., vol. 65, no. 6, pp. 2641-2656, 2017.

[14] Q. Sun, S. Han, C. L. I, and Z. Pan, "Energy efficiency optimization for fading MIMO non-orthogonal multiple access systems," IEEE Int. Conf. Commun., vol. 2015-Septe, no. 2014, pp. 26682673, 2015.

[15] Y. Zhang, Q. Yang, T. Zheng, H. Wang, Y. Ju, and Y. Meng, "Energy Efficiency Optimization in Cognitive Radio Inspired NonOrthogonal Multiple Access," IEEE Int. Symp. Pers. Indoor Mob. Radio Commun. PIMRC, vol. 27, 2016.

[16] L. Dai, B. Wang, Y. Yuan, S. Han, C. L. I, and Z. Wang, "Nonorthogonal multiple access for 5G: Solutions, challenges, opportunities, and future research trends," IEEE Commun. Mag., vol. 53, no. 9, pp. 74-81, 2015.

[17] M. F. Hanif, Z. Ding, T. Ratnarajah, S. Member, and G. K. Karagiannidis, "A Minorization-Maximization Method for Optimizing Sum Rate in the Downlink of Non-Orthogonal Multiple Access Systems," vol. 64, no. 1, pp. 76-88, 2016.

[18] M. Science, "On Nonlinear Fractional Programming Author ( s ) Werner Dinkelbach Source: Management Science, Vol . 13, No . 7 , Series A, Sciences ( Mar ., 1967 ), pp . 492-498 Published by: INFORMS Stable URL : http://www.jstor.org/stable/2627691," vol. 13, no. 7, pp. 492-498, 2018.

[19] Awasthi, Meenakshi, Nigam,M.J., Kumar Vijay, "Energy Efficient Sensing , Transmitting Time and Transmission Power for Cognitive Radio Networks,” in IEEE Indicon 2017., 2017. 